medium for transmission of force because he saw that every such mechanical transmission implied an infinite regress (the interaction of the particles of zether requiring another medium for their transmission, and so on ad infinitum): this is a mere guess, however, and not a likely one. What really worried Newton is that the density of the rether, on the evidence of the planetary motions, must be so small as to make it difficult to understand quantitatively how it could transmit gravitation. There can be no question, on the other hand, of Newton, as the authors would have it, abandoning the æther conception in despair and committing himself to the idea of action at a distance directly dependent on God's intervention. In their eagerness to defend this untenable view, they give to some of Newton's utterances an interpretation which I do not think these can bear when considered against their proper background.

My main reason for being so critical of what is, after all, a welcome addition to our knowledge of Newton's thought, has been to caution unwary readers, all too prone (as experience reveals) to imagine that the commentary exempts them from reading the text, or at least from independently making an effort to recapture, in studying these old documents, the struggle of a powerful mind towards truth and harmony. Yet, just in this effort lies the fascination of history.

L. Rosenfeld

\section{ADVANCES IN NUCLEAR SCIENCE}

\section{Annual Review of Nuclear Science}

Vol. 11. Edited by Emilio Segrè, in association with Gerhart Friedlander and Walter E. Moyerhof. Pp. vii +513. (Palo Alto, Calif.: Annual Reviews, Inc., 1961.) 7 dollars.

THE eleventh annual volume in this series of reviews is still very much the mixture as before. The editors have been sufficiently catholic in their selection of topics so that at least half the articles are of value to any scientist who has anything to do with nuclei. In this volume the depth of different articles differs rather markedly, but since they are meant to be topical rather than definitive this is to be expected. There are three reviews of fundamental particle and high-energy physics problems. "Statistical Methods in High Energy Physics", by Kretzsch. mar, is essentially a review of a theoretical physics technique. "Strong Interactions and Reactions of Hyperons and Heavy Mesons", by Morpurgo, does not include the recent surprising work on pion-pion resonances, and hence many of the conclusions may now be upset. The review of "Nucleon-Nucleon Elastic Scattering Theories", by Moravcsik and Noyes, is an excellent account of a most confused and difficult field. Though this is one of the most fundamental problems in all nuclear physics, the rate of progress toward a complete solution has been somewhat disappointing.

"Nuclear Orientation" (Roberts and Dabbs), "Neutron Capture Gamma Rays" (Bartholemew), and "Neutron Diffraction" (Wilkinson, Wollan and Koehler) are three excellent reviews of special fields of nuclear physics where the advances have been interesting and significant. Neutron diffraction is nowadays primarily a technique in the investigation of the solid and liquid state.

One of the most interesting features of these annual reviews are the articles on border-line fields which link several specializations. Arnold's review of "Nuclear
Effects of Cosmic Rays in Meteorites" is one of these, in which radioactivity analysis of extreme sensitivity can throw light on theories of cosmic rays and on the problem of the origin of meteorites. Another review of this type of great topical interest is "Detection of Nuclear Explosions", by Latter, Herbst and Watson. The major problem of the control of nuclear weapon tests has led to very interesting scientific investigations of the physical effects of such explosions on the environment. An article of much practical use to the nuclear physicist is Lindenbaum's "Shielding of High Energy Accelerators". The heavy-ion accelerators of the world are surveyed by Hubbard. "Isotope Effects in Chemical Reactions" by Weston is essentially physical chemistry of considerable importance to nuclear physicists. Libby's article on "Industrial Use of Isotopes" is rather an arbitrary selec. tion of some ways in which radioactive isotopes are used.

This volume also includes an index of the reviews in this series of the past five years, and should be of considerable appeal to those interested in nuclear science.

E. B. PaUl

\section{A DICTIONARY OF NUCLEAR PHYSICS AND TECHNOLOGY}

Dictionary of Nuclear Physics and Techno'ogy English-German-French-Russian. By Ralf Sube. Pp. 1,606. (Oxford, London, New York and Paris: Pergamon Press: Berlin: VEB Verlag Technik, 1961.) $400 s$.

TERR SUBE'S dictionary provides English, German, French and Russian equivalents of some 15,000 terms. His net has been cast fairly widely, and one might quarrel with the inclusion of quite a number of such entries as to act, crackling noise, optics and thick. The book consists of four sections, one for each language, in which the entries in the first column are in alphabetical order of that language, followed by three columns giving corresponding expressions in the other languages. In addition, each term has a letter and number derived from its English alphabetical position; the compiler explains that the purpose of this code is for use with complementary volumes to be published in other languages, but it could, presumably, just as well have been used to achieve a considerable reduction in the size of the present volume.

Gender and number are given where appropriate. Terms not recommended for standard usage have a cross-reference to a more acceptable term. American and British forms are generally distinguished. As usually happens in polyglot dictionaries, every term has its counterpart in all the other languages, and the user is left wondering how many of these are documented and how many provided by the compiler, especially when the 'equivalent' is a lengthy descriptive paraphrase. Apart from this, the dictionary is remarkably complete, accurate and free from errors. It is inevitable that a fow terms should have escaped attention, for example, flute instability, megaton, Mössbauer effect and whole-body counter; but they are a small minority. 'The compiler's English has occasionally led him into writing, for example, 'go critically' and including 'growth of grain' but not 'grain growth'; and in general the most complete explanations are given in the German column, so that the reader acquainted with German will be at 